\title{
Perceived relative distance on the ground affected by the selection of depth information
}

\author{
Jun Wu AND ZiJIANG J. He \\ University of Louisville, Louisville, Kentucky \\ AND \\ Teng Leng OoI \\ Pennsylvania College of Optometry, Elkins Park, Pennsylvania
}

\begin{abstract}
Our visual space does not appear to change when we scan or shift attention between locations. This appearance of stability implies that the depth information selection process is not crucial for constructing visual space. But we present evidence to the contrary. We focused on space perception in the intermediate distance, which depends on the integration of depth information on the ground. We propose a selection hypothesis that states that the integration process is influenced by where the depth information is selected. Specifically, the integration process inaccurately represents the ground when one samples depth information only from the far ground surface, instead of sequentially from the near to the far ground. To test this, observers matched the depth/length of a sagittal bar (test) to the width of a laterally oriented bar (reference) in three conditions in a full-cue environment that compelled the visual system to sample from different parts of the ground. These conditions had the lateral reference bar placed (1) adjacent to the test bar, (2) at the far ground, and (3) at the near ground. We found that the sagittal bar was perceived as shorter in conditions (1) and (2) than in Condition 3. This finding supports the selection hypothesis, since only Condition 3 led to more accurate ground surface integration/representation and less error in relative distance/depth perception. Also, we found that performances in all three conditions were similar in the dark, which has no depth information on the ground, indicating that the results cannot be attributed to asymmetric visual scanning but, rather, to differential information selection.
\end{abstract}

The ground theory of space perception claims a prominent role for the ground surface in localizing an object in the intermediate distance range (Gibson, 1950, 1979; Sedgwick, 1986), presumably due to the ecological significance of the ground surface (Gibson, 1950, 1979). Since its conception, the ground theory has found empirical supports from various laboratories. For example, it has been shown that the perceived distance of an object on the ground can be affected by the structure of the ground surface (Feria, Braunstein, \& Andersen, 2003; He, Wu, Ooi, Yarbrough, \& Wu, 2004; Sinai, Ooi, \& He, 1998; B. Wu, $\mathrm{He}$, \& Ooi, 2007; B. Wu, Ooi, \& He, 2004). A homogeneous ground surface, in conjunction with the object's angular declination, allows the object to be reliably localized (Ooi, Wu, \& He, 2001, 2006; Philbeck \& Loomis, 1997; J. Wu, He, \& Ooi, 2005). When the object is above the ground, the visual system can use the object's relative distance information with respect to the ground surface to localize the object (Madison, Thompson, Kersten, Shirley, \& Smits, 2001; Meng \& Sedgwick, 2001; Ni, Braunstein, \& Andersen, 2004; Ooi \& He, 2006; J. Wu, He, \& Ooi, 2004). Furthermore, a bias for using the ground surface is revealed in studies in which the visual system's per- formance has been measured under different types of large surface conditions - for example, ground-like versus ceiling-like surfaces (Bian, Braunstein, \& Andersen, 2005, 2006; McCarley \& He, 2000, 2001).

How does the visual system represent the ground surface for space perception? One possible way is based on the sequential surface integration process (SSIP; He et al., 2004; Ooi \& He, 2007; B. Wu et al., 2004). The SSIP hypothesis proposes that the visual system depends on both the external depth cues on the ground and the visual system's intrinsic bias to form the represented surface and that it constructs the ground surface representation from the near to the far distance. In a full-cue visual environment, the SSIP begins its operation by utilizing the reliable depth cues on the near ground surface (e.g., binocular disparity and motion parallax information) to form an accurate representation of the near ground surface. The near ground representation is then used as a template by the SSIP to integrate with the farther patches of ground surface, whose predominant depth information is the texture gradient. Thus, as the process extends from near to far, the SSIP is able to construct an accurate global ground surface representation. (If the stimulus condition is not

Z. J. He, zjhe@louisville.edu or T. L. Ooi, tlooi@pco.edu 
optimal for the SSIP, and depending on the type of information available, the visual system could rely on either the SSIP or other processes to form the global ground surface representation. The represented surface, however, will be less accurate.) In a viewing condition in which the ground surface is not visible (e.g., in complete darkness), the intrinsic bias of the visual system is treated as the ground surface. Our previous studies have shown that the intrinsic bias takes the form of an implicit surface that curves upward from the ground (Ooi et al., 2001, 2006; J. Wu, He, $\&$ Ooi, 2006). The local slant of the surface increases as the distance increases; but for our present purposes, we will assume that the shape of the intrinsic bias is approximately a plane surface with a constant slant. In a reducedcue environment with limited reliable near-depth cues, the visual system increases its reliance on the intrinsic bias to represent the ground surface, leading to an inaccurate ground surface representation (Sinai et al., 1998; B. Wu et al., 2007; B. Wu et al., 2004).

In the present article, to further understand how the visual system constructs the ground surface representation, we focus on the idea that a selection process influences the SSIP. This selection hypothesis argues that the SSIP, like many other perceptual processes, is affected by a selection process that samples the depth information on the ground surface. Confirming this hypothesis would mean that the ground surface representation and, thus, our space perception is affected by where the depth information on the ground is sampled. At first blush, this hypothesis appears somewhat counterintuitive, because it is inconsistent with the general view that our perceptual space is formed by an automatic process that does not depend on an attention selection process. As such, only a few studies have addressed the concern of whether perceived distance is affected by attention (Gogel \& Tietz, 1977).

In the present study, we tested a prediction of the selection hypothesis that the perceived relative distance/depth of a bar target on the ground surface depends on how the visual system selects the available depth information on the ground. We capitalized on the foreshortening phenomenon, in which the sagittal distance in the depth dimension (relative distance/depth) is underestimated, as compared with the lateral distance (width).
Our first experiment tested whether the selection process can affect the perceived foreshortening of a target on the ground in the full-cue environment (Figure 1). In the L-shaped condition (Figure 1A), an observer was asked to perceptually match the sagittal length (L) of the L-shaped target to its lateral width (W). It was expected that the observer would require the length of the L-shaped target to be longer to match its width; that is, the aspect ratio (W/L) of the matched L-shaped target would be smaller than 1 (Loomis \& Philbeck, 1999; Loomis, Philbeck, \& Zahorik, 2002; Sauer, Braunstein, Andersen, \& Bian, 2001). In addition, our previous studies have shown that the foreshortening of the L-shaped target is affected by the extent of the visible ground surface supporting the target (Ooi et al., 2006; B. Wu et al., 2004). For instance, foreshortening is larger when the visual field of view is limited to a small ground surface area $\left(<20^{\circ}\right)$ surrounding a distant L-shaped target than when the visual field of view is unrestricted (B. Wu et al., 2004). When only the limited visual information of the distant target and its vicinity, which excludes the near ground surface information, is sampled, the SSIP is prevented from forming an accurate template of the near ground representation for integration with the distant ground surface. Thus, the representation of the distant ground surface is heavily affected by the intrinsic bias of the visual system. In turn, the global representation of the ground surface has a slant error, and the foreshortening is increased.

From the foregoing, one could predict that less foreshortening would be found in the stimulus display in the near-reference condition (Figure 1B); that is, a larger matched ratio between the reference target and the test $\operatorname{target}(\mathrm{W} / \mathrm{L}$ ) would be found, as compared with that in the L-shaped condition (Figure 1A). Here, the observer had to match the sagittal length/depth of the test target to the lateral width of a reference target that was located on the near ground surface (this display modified the L-shaped target by spatially separating its limbs). The foreshortening effect was predicted to be less, because the observer could now scan the ground surface between the near-reference target and the test target to perform the matching task. As a result, the SSIP would be able to utilize the reliable near-depth cues on the near ground surface to form a more accurate ground surface representation.

The Four Stimulus Conditions in Experiments 1 and 2
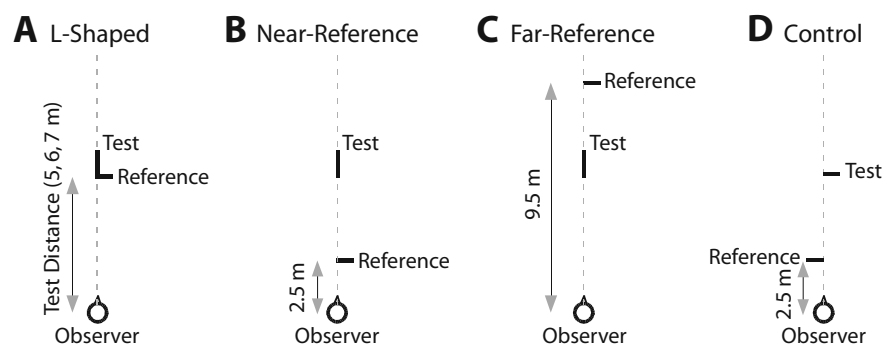

Figure 1. Illustration of the display conditions (top view). In panels A-C, the observers matched the sagittal length of the test target to the lateral width of the reference target. In panel D, they matched the lateral widths of both the test and the reference targets. 
The stimulus display in the far-reference condition (Figure 1C) reversed the stimulus design of the nearreference condition (Figure 1B) by placing the reference target beyond the test target on the far ground surface. As such, although the observer could scan a larger area of ground surface to view the test target and the far-reference target during the matching task, he/she would concentrate mainly on the ground surface area between the test and the reference targets. Less attention would be paid to the near ground surface, thus preventing the reliable near-depth cues from being sufficiently sampled to form an accurate global ground surface representation. Consequently, the selection hypothesis predicted that a smaller matched ratio (W/L) would be found in the far-reference condition (Figure 1C) than in the near-reference condition (Figure 1B).

In Experiment 1, it was assumed that the specific act of scanning and sampling of the depth information on a particular area of the ground surface would affect the accuracy of the ground surface representation and, hence, the foreshortening effect. The validity of this assumption requires that the impact of the scanning process on depth perception should be the same whether the reference target is at the near or the far ground surface. To evaluate this same-scanning-process hypothesis, our second experiment tested the same three display conditions in the dark, where the ground surface is not visible. The ground representation in the dark is contributed solely by the intrinsic bias, which can be approximated as a slant surface with its far end above the floor (Ooi et al., 2001, 2006). The foreshortening effect in the dark is dependent on the intrinsic bias, and not on the ground surface information (which is not visible). Thus, this predicts that the foreshortening in all three stimulus conditions will be the same - unless a scanning process that varies with scanning distance also influences the foreshortening effect.

The analysis and predictions above notwithstanding, it could still be argued that our favored empirical outcomes have an alternative explanation. It could be that the (lateral) reference target in the near-reference condition is simply estimated as longer than those in the far-reference and L-shaped conditions. Thus, for both Experiments 1 and 2 , to investigate the possibility of an increasing overestimation of the lateral width of the reference target with viewing distance, we tested our observers in a control condition in which both the test and the reference targets had the same (lateral) orientation (Figure 1D).

\section{EXPERIMENT 1 Full-Cue Environment}

\section{Method}

Participants. Eleven naive observers with informed consent and normal or corrected-to-normal visual acuity (at least 20/20) participated in the experiment. Of these, 7 observers with an average eye height of $157.9 \mathrm{~cm}$ were tested with the monocular-viewing protocol. The subsequent binocular-viewing protocol was conducted with 3 observers who had participated in the monocular-viewing protocol and 4 new observers who had not. Their average eye height was $159.0 \mathrm{~cm}$.

Displays and test site. The experiment was conducted on a large, homogeneous, horizontal grass field, from which the viewing dis- tance to the nearest tree or building was at least $20 \mathrm{~m}$ from the observer. A discreet, short wooden stick marked the observer's starting position. The lateral reference target in each of the four conditions was a white PVC pipe (1-in. diameter) with a fixed length of either $44 \mathrm{~cm}$ for the test trials or $30 \mathrm{~cm}$ for the catch trials. The variable length test target was made up of two white PVC pipes, with a 1-in.diameter pipe inserted into a 1.2-in.-diameter pipe. This allowed the pipes to slide over one another to obtain the desired length during a trial. The test target in all the conditions, including the sagittal limb of the L-shaped target in the L-shaped condition, was set at one of three test distances $(5,6$, or $7 \mathrm{~m})$ from the observer. Whereas the distance of the lateral reference target in the L-shaped condition changed with the test distance, it remained fixed at a selected viewing distance in the remaining three conditions (Figure 1).

Tasks and Procedure. The observers performed the matching task with both binocular- and monocular- (dominant eye) viewing protocols. Before a trial, the observer stood with his/her back facing the test scene, to allow the experimenter to set up the stimulus. Once set, the experimenter walked several meters away from the stimulus and instructed the observer to turn around to compare the sagittal length of the test target with the width of the reference target. To match the length and the width, the observer instructed the experimenter to adjust the length of the test target to an amount of length that was proportional to the perceived length - for example, decrease the length of the target by one fifth. He/she then turned his/her back to the test scene to allow the experimenter to make the adjustment, after which the experimenter again instructed the observer to turn around to make another judgment. This procedure was repeated (typically about three to five times) until the observer judged the length and width as equal. No feedback was given to the observer regarding the performance. On average, each trial took about 2-3 $\mathrm{min}$.

We ran two blocks of trials in the monocular-viewing protocol. The first block tested the L-shaped condition (Figure 1A) to investigate whether the observers could match the length and width of the targets on the basis of retinal image size. With this retinal image size response criterion, the observers were told to imagine taking a photograph of the L-shaped target and, on the basis of the perceived size on the photograph, to match the length and width. The order in which the target distances were tested was randomized.

The second block tested all four conditions in Figure 1 and was run after a 15-min break following the first test block. The observers now performed the task using the physical size response criterion (Loomis \& Philbeck, 1999; Loomis et al., 2002; Ooi et al., 2006; Sedgwick, 1986; B. Wu et al., 2004). The observer was told to imagine that he/she had walked up to the test target and looked down directly at it, in order to match the physical length and width. The order in which target distances and conditions were tested was randomized.

The four conditions in Figure 1 were also tested with the binocularviewing protocol, using procedures similar to those above and with the physical size response criterion. However, only one target distance $(6 \mathrm{~m})$ was tested.

The observers practiced the matching task according to the specified response criterion for 5-10 $\mathrm{min}$ before each test block. Before the experiment, the observers were informed that the length of the reference target would not be constant. Each block consisted of fourfifths test trials (reference target $=44 \mathrm{~cm}$ ) and one-fifth catch trials (reference target $=30 \mathrm{~cm}$ ). Only the data from the test trials were analyzed. Each target distance was tested twice.

\section{Results}

Monocular viewing. The filled triangles, squares, and circles in Figure 2A plot the average matched ratios (W/L) for the three stimulus conditions in Figures 1A, 1B, and $1 \mathrm{C}$, respectively, using the physical size response criterion. Overall, the matched ratios are smaller than 1 , indi- 


\section{Experiment 1:Full-Cue Environment}
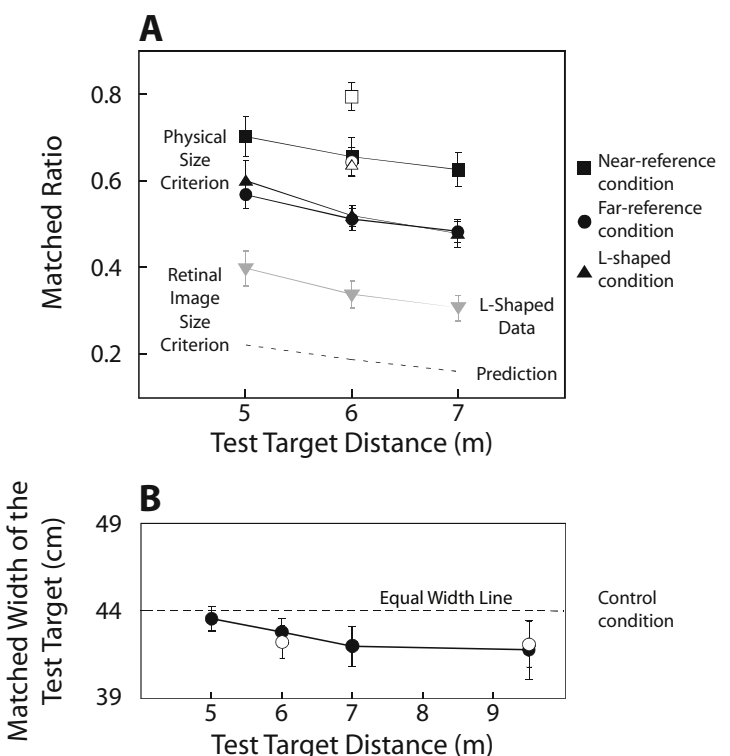

Control condition

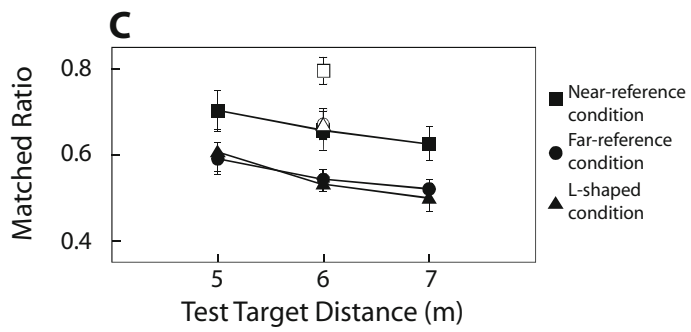

Figure 2. Average results of Experiment 1 performed in the full-cue environment. The filled and open symbols depict the average data with monocular and binocular viewing, respectively. (A) The average matched ratio (width of lateral bar/length of sagittal bar) is plotted as a function of the distance of the sagittal bar, for the conditions in Figures 1A-1C. The dash line predicts the matched ratios that would have been obtained had the observer's judgment been based solely on the retinal image size. (B) The average matched width of the lateral test bar with respect to the lateral reference bar $(44 \mathrm{~cm})$ at a viewing distance of $2.5 \mathrm{~m}$. (C) The corrected matched ratios for the data in panel $A$. See the text for details.

cating that a target's sagittal length is underestimated relative to its lateral width. The matched ratios decrease with increases in the test target distance $[F(2,12)=77.458$, $p<.00001$; two-way ANOVA with repeated measures], indicating that the relative underestimation of the sagittal length increases with increases in distance. A comparison among the three conditions reveals that the matched ratios are larger in the near-reference condition than in the far-reference condition [main effect of display condition, $F(1,6)=36.016, p<.001$; interaction effect (distance $\times$ display), $F(2,12)=0.214, p=.810$; two-way ANOVA with repeated measures] and in the L-shaped condition [main effect of display condition, $F(1,6)=72.11, p<.0005$; interaction effect (distance $\times$ display), $F(2,12)=1.539$, $p=.254$; two-way ANOVA with repeated measures].
Figure 2B plots the data for the control condition (Figure 1D), which tested the possibility that the lateral width of a distant target is overestimated relative to the width of a near target. Consistent with this, the average data show a tendency for the matched width to decrease with distance. However, a one-way ANOVA with repeated measures failed to reveal a significant effect of distance $[F(3,18)=1.826$, $p=.179]$. Nevertheless, to account for the residual contribution of an overestimation of the reference target to the matched ratios (the second factor) in the far-reference and L-shaped conditions (Figure 2A), we first calculated the ratio of the matched width of the test target at $9.5 \mathrm{~m}$ to the width of the reference target $(44 \mathrm{~cm}$ ) (Figure 2B). We then divided each of the matched ratio from the far-reference condition by the calculated ratio and plotted the results as the filled circles in Figure 2C. We also calculated the ratios at the 5-, 6-, and 7-m target distances in Figure 2B. We divided the matched ratios in the L-shaped condition by the calculated ratios at each corresponding test target distance (Figure 2A). The corrected matched ratios are plotted as filled triangles in Figure 2C. Clearly, the data in Figure 2C reveal that after correcting for the overestimation of the reference target, the matched ratios in the near-reference condition remain larger (i.e., smaller foreshortening) than those in the far-reference $[F(1,6)=16.950, p<.01 ; F(2,12)=$ $0.122, p=.886]$ and L-shaped $[F(1,6)=33.860, p<$ $.0025 ; F(2,12)=0.576, p=.577]$ conditions. These findings support the prediction of the selection hypothesis. Furthermore, note that the matched ratios for the far-reference and L-shaped conditions are similar, even though the former condition required the observer to scan a larger ground area. This suggests that at the far distance, the accuracy of the ground surface representation is not critically dependent on the size of the ground area sampled.

The gray triangles in Figure 2A plot the average matched ratios from the L-shaped condition based on the retinal image size response criterion. The matched ratios are significantly smaller than those obtained with the physical size criterion [filled triangles; $F(1,6)=20.871, p<.005$; $F(2,12)=1.087, p=.368]$. This finding suggests that the matched ratios can be influenced by the response criterion used during the experiment (Gilinsky, 1955). The dash line in Figure 2A indicates where the data would fall had the observers' performances been strictly based on the retinal image size response criterion. Clearly, the open triangles are above the dash line, indicating that even with explicit instructions, observers can only partially "ignore" the depth information on the ground surface (Burbeck, 1987; Gilinsky, 1955; McKee \& Welch, 1992).

Binocular viewing. The average results with binocular viewing (open symbols), using the physical size response criterion, have a trend similar to that with monocular viewing (filled symbols). As is shown in Figure 2A, the matched ratios are larger in the near-reference condition than in both the far-reference $[t(6)=8.92, p<$ $.001]$ and the L-shaped $[t(6)=10.34, p<.001]$ conditions. This trend remains the same after correcting for the overestimation of the reference target [Figure $2 \mathrm{C}$; near vs. far, $t(6)=6.78, p<.001$; near vs. L-shaped, $t(6)=6.81, p<.001]$. 


\section{EXPERIMENT 2 Dark Environment}

\section{Method}

Participants. Eight naive observers with informed consent and normal or corrected-to-normal visual acuity (at least 20/20) participated in the experiment. Three of the observers also participated in Experiment 1. The average eye height of the observers was $162.1 \mathrm{~cm}$.

Displays and test site. The targets were phosphorescent stripes with almost similar angular widths $(0.25 \mathrm{in}$. at $2.5 \mathrm{~m}$ and $1 \mathrm{in}$. at $9.5 \mathrm{~m})$. The lateral width of the reference target was either $30 \mathrm{~cm}$ (test trials) or $40 \mathrm{~cm}$ (catch trials). The four conditions from Experiment 1 were tested in a dark room. Although the targets were always placed on the floor, the observers were told that the targets could be either on or above the floor.

Procedure. The observers performed the matching task binocularly. Before the experiment, they spent $15-20$ min practicing the matching task in the lighted hallway outside the test room. The practice session was necessary particularly for the observers who had never performed the matching task using the physical size response criterion in the dark, because we have noticed in the past that without any training in using the physical size response criterion to perform the matching task, some observers can have a strong bias for using the retinal image size response criterion in the dark. Practicing in the light environment allowed the observers to easily notice the foreshortening phenomenon and become familiar with the physical size response criterion, which would lead to a stable performance.

After the practice, the observer was led to the test room without vision, to sit on a chair facing a wall near the starting position. A black curtain divided this waiting area (dark) and the test area (temporarily lighted). Once the phosphorescent targets were set up, the experimenter switched off the light in the test area and instructed the observer to stand, turn around, and step onto two phosphorescent elements on the floor (marking the starting position) facing the test area. The experimenter drew the black curtain open for the observer to begin the trial. The observer's task was to instruct the experimenter to adjust the sagittal length of the target to match the lateral width. Between instructions, the observer closed his/her eyes, and the experimenter drew the curtain closed to make the adjustments. This was repeated until the observer finished by judging the length and width to be equal. The observer received no feedback regarding the performance.

Before the proper experiment, the observer was given several practice trials in the dark. All four display conditions were tested in a single block, in which the trial order was randomized. Each trial was repeated twice. As in Experiment 1, the observer was informed that the length of the reference target was not constant. During the experiment, four fifths of the trials were test trials (reference target $=$ $30 \mathrm{~cm}$ ) and one fifth were catch trials (reference target $=40 \mathrm{~cm}$ ).

\section{Results}

We measured the matched ratios for all four conditions in Figure 1 in the dark, using the physical size response criterion. Figure $3 \mathrm{~A}$ plots the average matched ratios for the three test conditions in Figure 1. Overall, the matched ratios are smaller than those in the full-cue conditions in Experiment 1 (Figure 2A), indicating a stronger foreshortening when the ground surface is not visible in the dark, consistent with the results of our previous study (Ooi et al., 2006). But similar to the full-cue condition, the matched ratios decrease with target distance $[F(2,14)=34.135, p<.00005$; two-way repeated ANOVA with repeated measures].

Figure $3 \mathrm{~A}$ also reveals that the matched ratios in the near-reference condition (squares) are lower than those in the far-reference condition (circles) [main effect of display condition, $F(1,7)=18.289, p<.005$; interaction

\section{Experiment 2: Dark Environment}
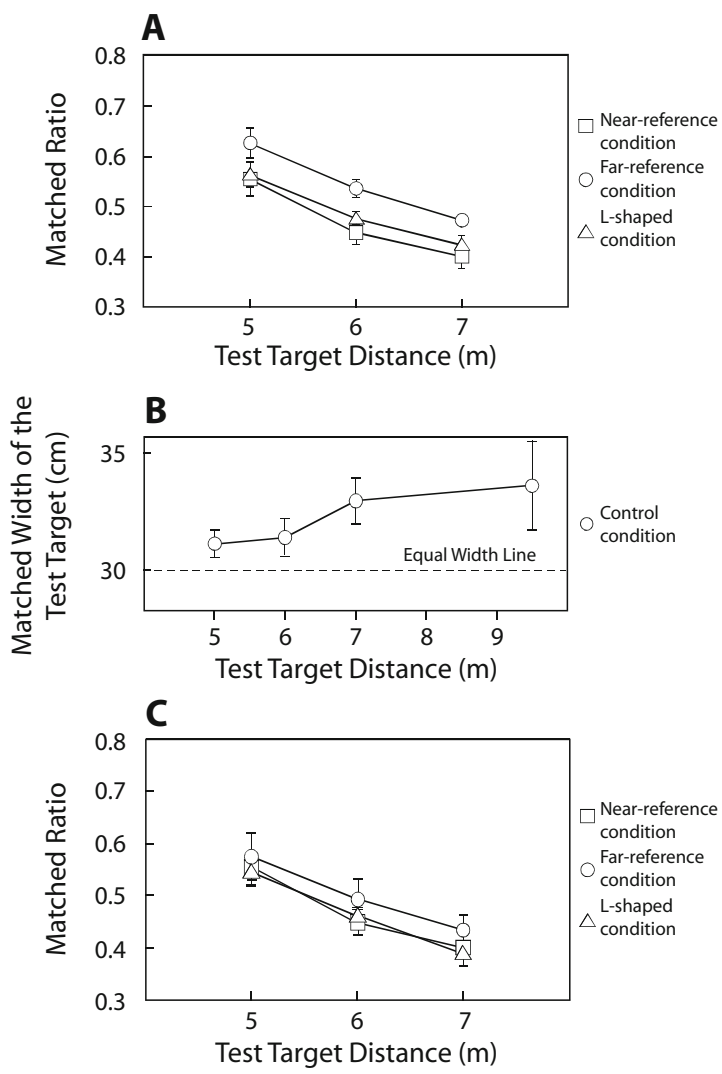

Figure 3. Average results of Experiment 2, performed in the dark environment (binocular viewing only). (A) The average matched ratio (width of lateral bar/length of sagittal bar) is plotted as a function of the distance of the sagittal bar, for the conditions in Figures 1A-1C. (B) The average matched width of the lateral test bar with respect to the lateral reference bar $(44 \mathrm{~cm})$ at a viewing distance of $2.5 \mathrm{~m}$. (C) The corrected matched ratios for the data in panel $\mathrm{A}$. See the text for details.

effect (distance $\times$ display), $F(2,14)=0.304, p=.743]$, and similar to the L-shaped condition $[F(1,7)=3.531$, $p=.1023 ; F(2,14)=0.639, p=.543]$. This differs from that in Experiment 1, where the matched ratios are significantly larger in the near-reference condition (Figure 2A).

As in Experiment 1, we addressed the possibility that misperceiving the lateral width of the reference target affected the matched ratios in the L-shaped and farreference conditions. The data from the control condition (Figure 3B) show that the observers progressively underestimated the lateral width with distance $[F(3,21)=$ $3.169, p<.05$; one-way ANOVA with repeated measures]. This trend is opposite to that in the full-cue environment (Figure 2B). Following the same procedure as that in Experiment 1 , we calculated the correction ratio (reference width/target length) from the data in Figure 3B and used them to correct the matched ratios in Figure 3A.

With correction, the matched ratios in all three conditions become similar [Figure 3C; main effect of display 
condition, $F(2,14)=0.140, p=.870$; interaction effect (distance $\times$ display), $F(4,28)=0.751, p=.565]$. This finding supports the same-scanning-process hypothesis, which predicts that the judged relative depth of a target is not affected by the idiosyncrasies of scanning itself when the matching task is performed. And given that the ground surface is not visible in the dark, the results in Figure 3 reinforce the conclusion of Experiment 1, which supports the selection hypothesis.

\section{DISCUSSION}

The selection hypothesis proposes that the SSIP, which represents the ground surface, is influenced by where depth information on the ground surface is sampled. To test the selection hypothesis, our study capitalized on the fact that the SSIP uses the depth information on the near ground to form an accurate ground surface representation (He et al., 2004; B. Wu et al., 2004). Supporting the hypothesis, we found that judged relative distance/depth on the ground is more accurate (less foreshortening) when the observer samples the large ground area that includes the near ground surface than when he/she samples the ground area that encompasses only the far ground surface.

The selection hypothesis provides an explanation for a puzzle in space perception. A number of studies have revealed that human observers can accurately judge egocentric distance on the ground, but not exocentric distance, which is underestimated (Beusman, 1998; Creem-Regehr, Willemsen, Gooch, \& Thompson, 2005; Loomis, DaSilva, Fujita, \& Fukusima, 1992; Loomis, DaSilva, Philbeck, \& Fukusima, 1996; Loomis et al., 2002; Norman, Crabtree, Clayton, \& Norman, 2005; Norman, Todd, Perotti, \& Tittle, 1996; Ooi et al., 2001, 2006; Philbeck, O'Leary, \& Lew, 2004; Rieser, Ashmead, Talor, \& Youngquist, 1990; Thomson, 1983; Toye, 1986; Wagner, 1985; B. Wu et al., 2004). A possible reason for the underestimation of exocentric distance, according to the selection hypothesis, is that the nature of the exocentric distance judgment task (e.g., the L-shaped task) encourages the visual system to attend locally to the ground surface information in the vicinity of the target, rather than to the global ground surface. On the other hand, the egocentric distance task (e.g., the blindfolded-walking task) encourages the observer to scan the ground from near his/her feet up to the target. Being able to sample the near ground information leads to an accurate ground surface representation and egocentric distance perception.

The notion of a selection process also adds a new dimension to our understanding of visual perception and cognition. It is often assumed that a process with unlimited capacity constructs our perceptual space. As such, information selection controlled by focal attention is not critical for the construction of the perceptual space but affects only the selection of the represented objects and the assessment of distances within the already constructed, stable perceptual space. Indeed, our everyday experience of a stable visual space appears to reinforce this assumption. After all, when we look around, or shift attention from one place to another, we are seldom aware of changes in the object distance or visual space. Yet the selection hypothesis predicts that our space perception changes when we look around. In particular, when one ignores the near ground surface, space perception becomes less veridical. Our present study, which relied on psychophysical measurements in the natural environment (Experiment 1), shows that perceived relative distance/ depth depends on which part of the ground surface information is sampled. Therefore, our study raises an interesting question for future research: Why do we not perceive objects "morphing" as we look around?

\section{AUTHOR NOTE}

This study was supported by Grant R01 EY014821 from the NIH to Z.J.H. and T.L.O. We thank Myron Braunstein, Harold Sedgwick, and an anonymous reviewer for their helpful comments on a draft of this article. Correspondence concerning this article should be addressed to either Z. J. He, Department of Psychological and Brain Sciences, University of Louisville, Louisville, KY 40292 (e-mail: zjhe@louisville.edu) or T. L. Ooi, Department of Basic Sciences, Pennsylvania College of Optometry, 8360 Old York Road, Elkins Park, PA 19027 (e-mail: tlooi@pco.edu).

\section{REFERENCES}

Beusman, J. M. H. (1998). Optic flow and the metric of the visual ground plane. Vision Research, 38, 1153-1170.

Bian, Z., Braunstein, M. L., \& Andersen, G. J. (2005). The ground dominance effect in the perception of 3-D layout. Perception \& Psychophysics, 67, 802-815.

Bian, Z., Braunstein, M. L., \& Andersen, G. J. (2006). The ground dominance effect in the perception of relative distance in 3-D scenes is mainly due to characteristics of the ground surface. Perception \& Psychophysics, 68, 1297-1309.

Burbeck, C. A. (1987). Locus of spatial-frequency discrimination. Journal of the Optical Society of America A, 4, 1807-1813.

Creem-Regehr, S. H., Willemsen, P., Gooch, A., \& Thompson, W. B. (2005). The influence of restricted viewing conditions on egocentric distance perception: Implications for real and virtual indoor environments. Perception, 34, 191-204.

Feria, C. S., Braunstein, M. L., \& Andersen, G. J. (2003). Judging distance across texture discontinuities. Perception, 32, 1423-1440.

GiBson, J. J. (1950). The perception of the visual world. Boston: Houghton Mifflin.

GiBson, J. J. (1979). The ecological approach to visual perception. Hillsdale, NJ: Erlbaum.

GILINSKY, A. S. (1955). The effect of attitude upon the perception of size. American Journal of Psychology, 68, 173-192.

Gogel, W. C., \& TiETZ, J. D. (1977). Eye fixation and attention as modifiers of perceived distance. Perceptual \& Motor Skills, 45, 343-362.

He, Z. J., Wu, B., OoI, T. L., Yarbrough, G., \& Wu, J. (2004). Judging egocentric distance on the ground: Occlusion and surface integration. Perception, 33, 789-806.

Loomis, J. M., DaSilva, J., Fujita, N., \& Fukusima, S. (1992). Visual space perception and visually directed action. Journal of Experimental Psychology: Human Perception \& Performance, 18, 906-921.

Loomis, J. M., DaSilva, J., Philbeck, J. W., \& Fukusima, S. (1996). Visual perception of location and distance. Current Directions in Psychological Science, 5, 72-77.

Loomis, J. M., \& PHILBEcK, J. W. (1999). Is the anisotropy of perceived 3-D shape invariant across scale? Perception \& Psychophysics, 61, 397-402.

Loomis, J. M., Philbeck, J. W., \& Zahorik, P. (2002). Dissociation of location and shape in visual space. Journal of Experimental Psychology: Human Perception \& Performance, 28, 1202-1212.

Madison, C., Thompson, W., Kersten, D., Shirley, P., \& Smits, B. (2001). Use of interreflection and shadow for surface contact. Perception \& Psychophysics, 63, 187-194.

McCarley, J. S., \& He, Z. J. (2000). Asymmetry in 3-D perceptual 
organization: Ground-like surface superior to ceiling-like surface. Perception \& Psychophysics, 62, 540-549.

McCarley, J. S., \& He, Z. J. (2001). Sequential priming of 3-D perceptual organization. Perception \& Psychophysics, 63, 195-208.

McKeE, S. P., \& Welch, L. (1992). The precision of size constancy. Vision Research, 32, 1447-1460.

Meng, J. C., \& SeDGWICK, H. A. (2001). Distance perception mediated through nested contact relations among surfaces. Perception \& Psychophysics, 63, 1-15.

Ni, R., Braunstein, M. L., \& Andersen, G. J. (2004). Perception of scene layout from optical contact, shadows and motion. Perception, 33, 1305-1318.

Norman, J. F., Crabtree, C. E., Clayton, A. M., \& Norman, H. F. (2005). The perception of distances and spatial relationships in natural outdoor environments. Perception, 34, 1315-1324.

Norman, J. F., Todd, J. T., Perotti, V. J., \& Tittle, J. S. (1996). The visual perception of three-dimensional length. Journal of Experimental Psychology: Human Perception \& Performance, 22, 173-186.

OoI, T. L., \& HE, Z. J. (2006). Localizing suspended objects in the intermediate distance range ( $>2$ meters) by observers with normal and abnormal binocular vision [Abstract]. Journal of Vision, 6, 853a. Available at journalofvision.org/6/6/853.

OoI, T. L., \& HE, Z. J. (2007). A distance judgment function based on space perception mechanisms: Revisiting Gilinsky's (1951) equation. Psychological Review, 114, 441-454.

OoI, T. L., Wu, B., \& He, Z. J. (2001). Distance determined by the angular declination below the horizon. Nature, 414, 197-200.

OoI, T. L., Wu, B., \& He, Z. J. (2006). Perceptual space in the dark affected by the intrinsic bias of the visual system. Perception, 35, 605-624.

Philbeck, J. W., \& Loomis, J. M. (1997). Comparison of two indicators of perceived egocentric distance under full-cue and reduced-cue conditions. Journal of Experimental Psychology: Human Perception \& Performance, 23, 72-85.

Philbeck, J. W., O'Leary, S., \& LeW, A. L. B. (2004). Large errors, but no depth compression, in walked indications of exocentric extent. Perception \& Psychophysics, 66, 377-391.

Rieser, J. J., Ashmead, D., Talor, C., \& Youngquist, G. (1990). Vi- sual perception and the guidance of locomotion without vision to previously seen targets. Perception, 19, 675-689.

Sauer, C. W., Braunstein, M. L., Andersen, G. J., \& Bian, Z. (2001). Judged shape of ground plane regions in realistic 3-D scenes [Abstract]. Journal of Vision, 1, 383a. Retrieved Oct. 2, 2006, from journalofvision.org/1/3/41.

SeDGwick, H. A. (1986). Space perception. In K. R. Boff, L. Kaufman, \& J. P. Thomas (Eds.), Handbook of perception and human performance (pp. 21.1-21.57). New York: Wiley.

SinAI, M. J., OoI, T. L., \& He, Z. J. (1998). Terrain influences the accurate judgement of distance. Nature, 395, 497-500.

Thomson, J. A. (1983). Is continuous visual monitoring necessary in visually guided locomotion? Journal of Experimental Psychology: Human Perception \& Performance, 9, 427-443.

ToYe, R. C. (1986). The effect of viewing position on the perceived layout of space. Perception \& Psychophysics, 40, 85-92.

Wagner, M. (1985). The metric of visual space. Perception \& Psychophysics, 38, 483-495.

Wu, B., He, Z. J., \& OoI, T. L. (2007). The linear perspective information in ground surface representation and distance judgment. Perception \& Psychophysics, 69, 654-672.

Wu, B., OoI, T. L., \& HE, Z. J. (2004). Perceiving distance accurately by a directional process of integrating ground information. Nature, 428, $73-77$

Wu, J., HE, Z. J., \& OoI, T. L. (2004). Stimulus duration and binocular disparity factors in representing the ground surface and localizing object in the intermediate distance range [Abstract]. Journal of Vision, 4, 21a. Available at journalofvision.org/4/8/21/.

Wu, J., HE, Z. J., \& OoI, T. L. (2005). Visually perceived eye level and horizontal midline of the body trunk influenced by optic flow. Perception, 34, 1045-1060.

Wu, J., HE, Z. J., \& OoI, T. L. (2006). The slant of the visual system's intrinsic bias in space perception and its contribution to ground surface representation [Abstract]. Journal of Vision, 6, 730a. Available at journalofvision.org/6/6/730/.

(Manuscript received March 27, 2007;

revision accepted for publication December 17, 2007.) 